NASA/TM-2003-212142

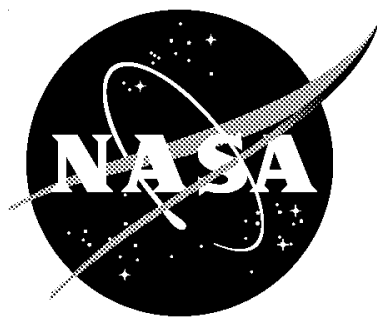

An Efficient and Robust Singular Value Method for Star Pattern Recognition and Attitude Determination

Jer-Nan Juang

Langley Research Center, Hampton, Virginia

Hye Young Kim and John L. Junkins

Texas A\&M University, College Station, Texas 


\section{The NASA STI Program Office ... in Profile}

Since its founding, NASA has been dedicated to the advancement of aeronautics and space science. The NASA Scientific and Technical Information (STI) Program Office plays a key part in helping NASA maintain this important role.

The NASA STI Program Office is operated by Langley Research Center, the lead center for NASA's scientific and technical information. The NASA STI Program Office provides access to the NASA STI Database, the largest collection of aeronautical and space science STI in the world. The Program Office is also NASA's institutional mechanism for disseminating the results of its research and development activities. These results are published by NASA in the NASA STI Report Series, which includes the following report types:

- TECHNICAL PUBLICATION. Reports of completed research or a major significant phase of research that present the results of NASA programs and include extensive data or theoretical analysis. Includes compilations of significant scientific and technical data and information deemed to be of continuing reference value. NASA counterpart of peer-reviewed formal professional papers, but having less stringent limitations on manuscript length and extent of graphic presentations.

- TECHNICAL MEMORANDUM. Scientific and technical findings that are preliminary or of specialized interest, e.g., quick release reports, working papers, and bibliographies that contain minimal annotation. Does not contain extensive analysis.

- CONTRACTOR REPORT. Scientific and technical findings by NASA-sponsored contractors and grantees.
- CONFERENCE PUBLICATION. Collected papers from scientific and technical conferences, symposia, seminars, or other meetings sponsored or co-sponsored by NASA.

- special publication. Scientific, technical, or historical information from NASA programs, projects, and missions, often concerned with subjects having substantial public interest.

- TECHNICAL TRANSLATION. Englishlanguage translations of foreign scientific and technical material pertinent to NASA's mission.

Specialized services that complement the STI Program Office's diverse offerings include creating custom thesauri, building customized databases, organizing and publishing research results ... even providing videos.

For more information about the NASA STI Program Office, see the following:

- Access the NASA STI Program Home Page at http://www.stinasa.gov

- E-mail your question via the Internet to help@sti.nasa.gov

- Fax your question to the NASA STI Help Desk at (301) 621-0134

- Phone the NASA STI Help Desk at (301) 621-0390

- Write to:

NASA STI Help Desk NASA Center for AeroSpace Information 7121 Standard Drive Hanover, MD 21076-1320 
NASA/TM-2003-212142

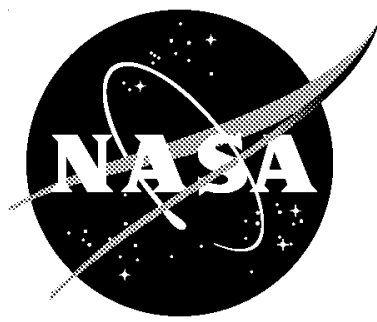

\section{An Efficient and Robust Singular Value Method for Star Pattern Recognition and Attitude Determination}

Jer-Nan Juang

Langley Research Center, Hampton, Virginia

Hye Young Kim and John L. Junkins

Texas A\&M University, College Station, Texas

National Aeronautics and

Space Administration

Langley Research Center

Hampton, Virginia 23681-2199

January 2003 
Available from:

NASA Center for AeroSpace Information (CASI)

7121 Standard Drive

Hanover, MD 21076-1320

(301) 621-0390
National Technical Information Service (NTIS)

5285 Port Royal Road

Springfield, VA 22161-2171

(703) $605-6000$ 


\title{
An Efficient and Robust Singular Value Method for Star Pattern Recognition and Attitude Determination
}

\author{
Jer-Nan Juang ${ }^{1}$ \\ NASA Langley Research Center \\ Hampton, VA 23681
}

\author{
Hye-Young $\mathrm{Kim}^{2}$ and John L. Junkins ${ }^{3}$ \\ Texas A\&M University \\ College Station, TX 77843
}

\begin{abstract}
A new star pattern recognition method is developed using singular value decomposition of a measured unit column vector matrix in a measurement frame and the corresponding cataloged vector matrix in a reference frame. It is shown that singular values and right singular vectors are invariant with respect to coordinate transformation and robust under uncertainty. One advantage of singular value comparison is that a pairing process for individual measured and cataloged stars is not necessary, and the attitude estimation and pattern recognition process are not separated. An associated method for mission catalog design is introduced and simulation results are presented.
\end{abstract}

\section{Introduction}

Star cameras are among the most attractive attitude sensors because they provide three-axis attitude information with high accuracy. Star pattern recognition is an integrated part of star cameras. It is based on the invariant properties from coordinate transformation. For the past two decades, many of the invariant properties, such as angular separation, brightness of star, shape of triangles with vertices of star, and constellations, have been utilized for star identification. ${ }^{1-9}$ In practice, one of the challenges of using these star trackers arises when initializing sensors and/or recovering them from sudden failure is to efficiently solve the "lost-in-space" problem. Even though a-priori attitude estimation is not necessary for a star tracker, these initializing problems without a-priori attitude information tend to require intensive computer storage and

\footnotetext{
${ }^{1}$ Principal Scientist, Structural Dynamics

${ }^{2}$ Graduate Student, Department of Aerospace Engineering

${ }^{3}$ Distinguish University Professor, Department of Aerospace Engineering
} 
computation time; For example, one popular pattern recognition method uses triplet angular separation matching, which is discussed by Gottlieb. ${ }^{10}$ Mortari $^{8}$ introduces a Search-Less algorithm, where angular separation is indexed as integer, and a procedure is introduced to directly access all possible star pairs that correspond to a given measured star pair. Besides angular separation comparisons, many star pattern recognition methods are introduced, such as star constellation matching, triangle matching, stochastic approach, and other more difficult to characterize methods based on fuzzy logic, neural networks, and so on. Usually, the brightness of a star is used as a secondary filter, because position measurement is usually the most accurate data available. While measurement of absolute star magnitude is usually inaccurately correlated with cataloged visual magnitude or catalog-based predictions of instrument magnitude, the relative magnitudes of stars imaged by the star camera are usually more nearly correlated to predictions. We can use magnitude information implicitly by ranking their relative measured magnitude and use this as secondary information.

In the study of the attitude determination problem, the most robust estimators minimizing Wahba's loss function are Davenport's q method ${ }^{11-15}$ and the Singular Value Decomposition (SVD) method by Markley. ${ }^{16}$ The q method, which computes the optimal quaternion as the eigenvector of a symmetric $4 \times 4$ matrix with the largest eigenvalue, is faster and produces more convenient output. The SVD solution is completely equivalent to the original solution by Farrell and Stuelpnagel. ${ }^{17}$ The difference is that computing the SVD is one of the most robust numerical algorithms.

In this study, a simple and inexpensive attitude initialization method for solving the lost-in-space problem is developed by introducing other unique, transformation invariant properties. These invariant properties are derived from the singular value decomposition of the reference star vectors and their corresponding vectors measured in the camera axes used for both star pattern recognition and attitude determination. A new mission catalog is generated, and attitude is estimated without the necessity of individual star pairing. 


\section{Singular Value Decomposition Method}

Let $v_{i}$ and $w_{i}(i=1, \ldots, N)$ be the reference (cataloged) unit vectors and their corresponding vectors measured in the camera axes. These vectors satisfy

$$
W=C V
$$

where column vector matrices $W$ and $V$ are defined as

$$
\begin{aligned}
& W=\left[\begin{array}{llll}
w_{1} & w_{2} & \cdots & w_{N}
\end{array}\right] \\
& V=\left[\begin{array}{llll}
v_{1} & v_{2} & \cdots & v_{N}
\end{array}\right]
\end{aligned}
$$

and $C$ is the direction cosine matrix (the unknown rotation matrix associated with the spacecraft angular position) such that

$$
C^{T} C=I
$$

or

$$
C^{T}=C^{-1}
$$

By Singular Value Decomposition (SVD), $W$ and $V$ are factorized as

$$
\begin{aligned}
& V=P_{v} \Sigma_{v} Q_{v}{ }^{T}=\sum_{i=1}^{3} p_{v_{i}} \sigma_{v i} q_{v_{i}}{ }^{T} \\
& W=P_{w} \Sigma_{w} Q_{w}{ }^{T}=\sum_{i=1}^{3} p_{w i} \sigma_{w i} q_{w i}{ }^{T}
\end{aligned}
$$

where $P_{v}$ and $P_{w}$ are $3 \times 3$ orthogonal matrices of left singular vectors $p_{v i}$ and $p_{w i},(i=1$, 2,3), $Q_{v}$ and $Q_{w}$ are $N \times N$ orthogonal matrices of right singular vectors $q_{v i}$ and $q_{w i},(i=1$, $2, \ldots, \mathrm{N})$, and $\Sigma_{v}$ and $\Sigma_{w}$ are $3 \times N$ diagonal matrices with singular values $\sigma_{v i}$ and $\sigma_{w i},(i=$ $1,2,3$ ), of $V$ and $W$. For any number of $N \geq 3$ distinct vectors, there are exactly 3 nonzero singular values and 3 left and right distinct singular vectors that may be used to uniquely parameterize the matrices $W$ and $V$.

\section{Invariant Singular Values}

Post-multiplying Eq. (1) by $W^{T}$, we obtain 


$$
W W^{T}=C V V^{T} C^{T}
$$

A similarity transformation is evident in Eq. (5) between the positive definite symmetric matrix $W W^{T}$ and the positive definite symmetric matrix $V V^{T}$ because $C$ is a unitary matrix and thus $C^{-1}=C^{T}$. Substituting Eqs. (4a) and (4b) into Eq. (5) yields

$$
\begin{aligned}
W W^{T} & =P_{w} \Sigma_{w} Q_{w}{ }^{T} Q_{w} \Sigma_{w}{ }^{T} P_{w}{ }^{T}=P_{w} \tilde{S}_{w} P_{w}{ }^{T} \\
& =C V V^{T} C^{T}=C P_{v} \Sigma_{v} Q_{v}^{T} Q_{v} \Sigma_{v}{ }^{T} P_{v}^{T} C^{T}=C P_{v} \tilde{S}_{v} P_{v}^{T} C^{T}
\end{aligned}
$$

or

$$
P_{w} \tilde{S}_{w} P_{w}^{T}=C P_{v} \tilde{S}_{v} P_{v}^{T} C^{T}
$$

where $\tilde{S}_{w}$ and $\tilde{S}_{v}$ are $3 \times 3$ diagonal matrices with eigenvalues $\left(\sigma_{w 1}{ }^{2},{\sigma_{w 2}}^{2}, \sigma_{w 3}{ }^{2}\right)$ and $\left(\sigma_{v 1}^{2}, \sigma_{v 2}^{2}, \sigma_{v 3}^{2}\right)$, respectively, given by

$$
\tilde{S}_{w}=\sum_{w} \Sigma_{w}{ }^{T} \text { and } \tilde{S}_{v}=\sum_{v} \Sigma_{v}{ }^{T}
$$

Since eigenvalues are preserved with a similarity transformation, $W W^{T}$ and $V V^{T}$ in Eq. (5) have the same eigenvalues, so

$$
\tilde{S}_{w}=\tilde{S}_{v}
$$

or

$$
\sigma_{w 1}^{2}=\sigma_{v 1}^{2}, \quad \sigma_{w 2}^{2}=\sigma_{v 2}^{2}, \quad \sigma_{w 3}^{2}=\sigma_{v 3}^{2}
$$

It implies that the singular values of $W$ and $V$ must satisfy

$$
\sigma_{w 1}=\sigma_{v 1}=\sigma_{1}, \quad \sigma_{w 2}=\sigma_{v 2}=\sigma_{2}, \quad \sigma_{w 3}=\sigma_{v 3}=\sigma_{3}
$$

where $\sigma_{1}, \sigma_{2}$, and $\sigma_{3}$ are introduced to signify their equality. In other words, $W$ and $V$ clearly have identical singular values. This result is consistent with well-known truths about the SVD; the singular values of a general matrix $M$ are the eigenvalues of the squared symmetric matrices $M M^{T}$ or $M^{T} M$, and the eigenvectors of $M M^{T}$ and $M^{T} M$ are the corresponding singular vectors. 


\section{Invariant Right Singular Vectors}

Pre-multiplying Eq. (1) by $W^{T}$ and using the identity of Eq. (3a), one obtains

$$
W^{T} W=V^{T} C^{T} C V=V^{T} V
$$

Using Eq. (4) for the SVD of $W$ and $V$, and Eq. (10) for equal singular values of $W$ and $V$, the matrices $W^{T} W$ and $V^{T} V$ can be written as

$$
\begin{aligned}
& W^{T} W=Q_{w} \Sigma^{T} P_{w}^{T} P_{w} \Sigma Q_{w}^{T}=Q_{w} \Sigma^{T} \Sigma Q_{w}^{T}=Q_{w} S Q_{w}^{T} \\
& V^{T} V=Q_{v} \Sigma^{T} P_{v}^{T} P_{v} \Sigma_{v} Q_{v}^{T}=Q_{v} \Sigma^{T} \Sigma Q_{v}^{T}=Q_{v} S Q_{v}^{T}
\end{aligned}
$$

where $S$ is an $\mathrm{N} \times \mathrm{N}$ diagonal matrix with three non-zero eigenvalues $\left(\sigma_{1}^{2}, \sigma_{2}^{2}, \sigma_{3}^{2}\right)$. Equation (13a) and (13b) become

$$
\begin{aligned}
& W^{T} W=\sigma_{1}^{2} q_{w 1} q_{w 1}^{T}+\sigma_{2}^{2} q_{w 2} q_{w 2}^{T}+\sigma_{3}^{2} q_{w 3} q_{w 3}^{T} \\
& V^{T} V=\sigma_{1}^{2} q_{v 1} q_{v 1}^{T}+\sigma_{2}^{2} q_{v 2} q_{v 2}^{T}+\sigma_{3}^{2} q_{v 3} q_{v 3}^{T}
\end{aligned}
$$

Assume that the singular values of $W$ and $V$ are distinct. Two positive, semi-definite matrices with distinct eigenvalues are identical if and only if their nonzero eigenvalues and orthonormal eigenvectors are identical. Note that each orthonormal vector is unique, except for its sign. Since $q_{w i}$ and $q_{v i}$ are orthonormal eigenvectors of $W^{T} W$ and $V^{T} V$, respectively, they must satisfy

$$
q_{w i}= \pm q_{v i}, \quad i=1,2,3
$$

Equation (15) can be rewritten as

$$
Q_{w}(:, 1: 3)=Q_{v}(:, 1: 3) L
$$

where $L$ is $3 \times 3$ diagonal matrix, given by

$$
L=\operatorname{diag}\left[\operatorname{sign}\left(q_{w 1}^{T} q_{v 1}\right) \quad \operatorname{sign}\left(q_{w 2}^{T} q_{v 2}\right) \quad \operatorname{sign}\left(q_{w 3}^{T} q_{v 3}\right)\right]
$$

Equations (11) and (16) represent invariant properties of $W$ and $V$ with any unitary coordinate transformation. Here we assume that both star sequences from the reference map and the measurements are in the same order. The assumption is possible by ordering 
the stars with their degree of brightness. Nevertheless, confusion may arise when two stars have the same brightness and thus some rows in $q_{w i}$ and $q_{v i}$ may not be consistent, unless we take careful steps to minimize the probability of this event. Since more than 4 measured stars are usually in the field of view, we can always choose the stars in ascending order with the brightest star being the first, and the faintest being the fourth. We can delete measured stars that are not separated by at least a relative magnitude increment of 0.2 (this has been found to be sufficient in virtually all cases). Obviously, analogous steps must be taken in creating the catalog that will be used to extract the corresponding reference vectors. Since we are only interested in the sign of the two vectors $q_{w i}$ and $q_{v i}$, we may compare the maximum value of $q_{w i}$ and the maximum value of $q_{v i}$ and take the sign of the resultant product to replace the $\operatorname{sign}\left(q_{w i}^{T} q_{v i}\right)$ in Eq. (17). The sign is needed for final computation of the direct cosine matrix $C$.

\section{Computation of Direct Cosine Matrix}

Consider column exchanges of $W$ by an $\mathrm{N} \times \mathrm{N}$ permutation matrix $K$

$$
\hat{W}=W K
$$

where

$$
K K^{T}=I
$$

Post-multiplying matrix Eq. (18) by $\hat{W}^{T}$ gives

$$
\hat{W} \hat{W}^{T}=W K K^{T} W^{T}=W W^{T}=\sum_{i=1}^{N} w_{i} w_{i}^{T}
$$

The sum of the outer product of column vectors of $W$ is preserved with column exchanges of $W$, implying that the singular values and the left singular vectors of $\hat{W}$ remain unchanged. The same statement is also true for the reference vectors in $V$. This means that individual vector paring process is not necessary for the pattern recognition, if the measurement set and reference set has one-to-one relationship, given by

$$
\hat{W}=W K=C V
$$

Note that the singular values of $\hat{W}$ and $V$ are identical. 
Unlike other pattern recognition methods, SVD pattern recognition method yields attitude estimate directly. In Eq. (7), $p_{w i}$ and $p_{v i}$ should satisfy

$$
p_{w i}= \pm C p_{v_{i}}, \quad i=1,2,3
$$

Equation (4a) indicates that the sign of $q_{v i}$ in Eq. (15) should be consistent with the sign of $p_{v i}$ in Eq. (22). Let $P_{w}$ be the matrix of $p_{w i}$. Equation (7) thus gives that

$$
P_{w}=C P_{v} L
$$

where $L$ is the $3 \times 3$ matrix shown in Eq. (17). The orthogonal attitude matrix is estimated as

$$
C=P_{w}\left(P_{v} L\right)^{T}
$$

In the ideal case, the two matrices $W$ and $V$ have identical singular values. In reality, the measurement vectors are corrupted by several error sources, including CCD precision, bias, and sensor misalignment. Under these uncertainties, we need to check if these singular values are still unique or not.

\section{Physical Interpretation}

To gain insight about singular values, let us consider the physical meaning of SVD of the matrix $W$. Define a matrix $A$ as

$$
A=W W^{T}=w_{1} w_{1}^{T}+w_{2} w_{2}{ }^{T}+w_{3} w_{3}^{T}+\cdots+w_{N} w_{N}{ }^{T}
$$

Then, the square of the maximum singular value of $W$ is obtained from

$$
\sigma_{\max }^{2}=\max _{x \neq 0} \frac{\|A x\|}{\|x\|}
$$

Assume that $x$ is on the unit circle, then

$$
\sigma_{\max }^{2}=\max _{\substack{x \neq 0 \\\|x\|=1}}\|A x\|
$$

where

$$
A x=W W^{T} x=w_{1} w_{1}^{T} x+w_{2} w_{2}{ }^{T} x+\cdots+w_{N} w_{N}{ }^{T} x
$$


For simplicity, consider the case of $\mathrm{N}=1$. The outer product of $w_{1}$ becomes the projection of $x$ onto $w_{1}$, as shown in Figure. 1. The projection of $x$ onto $w_{1}$ is given by

$$
p=w_{1} w_{1}^{T} x
$$

The maximum of $p$ happens when $x$ is parallel to $w_{1}$. Similarly, for $N>1$, the problem is to find the unit vector $x$ so that the sum of projections of $x$ onto each column vectors $w_{i}$ is maximized. Then, $x$ becomes the left singular vector associated with the maximum singular value, and $\|A x\|$ is the square of the maximum singular value of $W$. The square of the minimum singular value of $W$ represents the minimum value of $\|A y\|$ when the sum of projections of the unit vector $y$ (perpendicular to $x$ ) onto each column vector $w$ is minimized. Finally, the square of the intermediate singular value of $W$ represents the projection of the unit vector $z$, perpendicular to the plane generated by $x$ and $y$, onto each column vector. These three singular values and three left singular vectors represent $3-D$ ellipsoid with three principle axes of singular vectors and three semi-axis lengths of singular values. If the unit vector is not perfectly measured while the uncertainty is less than prescribed tolerance, these projections may not change drastically like criterion of any other pattern recognition method. So, under some degree of measurement uncertainty, these singular values represent a unique property of the unit column vector matrix that is very useful for star pattern recognition.

\section{Pattern Recogntion and MIssion Catalog Generation}

The SVD method can be easily applied to initializing the attitude of a star tracker and the lost-in-space case. Conventional star trackers commonly use angular separation or length ratio of a triangle for pattern recognition. Assume that the mission catalog contains $M$ guide stars. In the lost-in-space case, the angular separation method requires $M(M-1) / 2$ comparisons for each angular separation in the measurement set while the triangle method requires $M(M-1)(M-2) / 6$ comparisons for each triangle. Although, by pre-processing, which eliminates pairs with larger angular separation than the FOV diameter, the comparison number is still huge and time-consuming and also yields tremendous ambiguities. The SVD method, with judicious building of a mission catalog, will become the most reliable and fast attitude initializing method. 
A reference star catalog usually includes right ascension (RA), declination (DEC), and visual magnitude $(\mathrm{Mv})$ of stars. Some pattern recognition methods use only position information, whereas others use magnitude information and position information. In the star camera, star light magnitude information is not as accurate as position information. It depends on the specific camera and time. Moreover, the conversion of instrumental magnitude to visual magnitude is much more difficult. So, direct use of magnitude information is not desirable and has been used as a secondary filter. However, the relative magnitude between stars in the star camera is more accurate than magnitude itself, since visual magnitude of a star is proportional to the pixel intensity and area occupied by starlight, which allows us to rank the stars by their relative magnitude. The number of stars increases exponentially as magnitude increases; or as magnitude increases, magnitude ambiguity also increases. To eliminate the ambiguity of rank, we can use the 4 brightest stars from each star image. In this study, we consider an $8^{\circ} \times 8^{\circ}$ FOV star camera, which can capture enough number of stars to meet most attitude estimation precision. Our mission catalog will be constructed considering these aspects. Stars of most mission catalogs are filtered by a pre-specified visual magnitude limit considering the size of the FOV.

If the magnitude limit is small, there is a non-zero possibility that the catalog has holes. When the star camera looks at these holes, no guide star is found. On the other hand, if the magnitude limit is large, there exists a lot of ambiguity for pattern recognition, and computer storage is wasted. In this study, we did not set any specific magnitude limit, but generated a mission catalog which does not have any hole for an $8^{\circ}$ $\times 8^{\circ}$ circular FOV and at least 4 stars in that FOV. In fact, stars in the celestial sphere are not evenly distributed. Moreover, to find evenly distributed points in the sphere for a small FOV is impossible. Instead of finding the evenly distributed points, we can use modern computational power. Since the generation of mission catalog is pre-process, this does not harm the speed and storage for a real mission; and the final mission catalog is very slim and neat, which will be shown later.

Let us consider brighter stars than $7.3 \mathrm{Mv}$, where no hole for an $8^{\circ} \times 8^{\circ}$ circular FOV exists. Distribution of reference catalog stars is shown in Figure. 2. First, RA and DEC for each star are converted to a direction cosine vector. Then, too close stars and 
double stars are eliminated using the inner product of direction cosine vectors. Consider each direction cosine vector as the boresight direction, and filter adjacent stars so that angular separation from the boresight direction is less than $4^{\circ}$. Adjacent stars, including the boresight direction star, are sorted by their visual magnitude in ascending order; then, the 4 brighter stars are chosen, considering the same visual magnitude of 4 th-brightest stars and 5th-brightest stars (possibly more than 2). All $M$ boresight directions have 4 adjacent stars at $7.3 \mathrm{Mv}$; or there is no star which does not have 4 adjacent stars. Although $M$ is a huge number, it is about twice bigger than real distinctive sets by duplication. Duplication is arisen in the case of Figure. 3. The letter $(a, b, \ldots)$ represents the name of a star, and the number is the rank of brightness of each star. Consider $d$ as the boresight direction. Temporary adjacent stars are encircled by circular FOV $D$ and sorted by their visual magnitude and ranked. Then, the 4 brightest stars are the set of $[b, d, e, f]$. Next, consider the star $a$ as the boresight direction, and temporary adjacent stars are encircled by circular FOV $A$. By the same procedure, adjacent stars for the star $a$ become $[b, d, e, f]$. Thus, the set $[b, d, e, f]$ is duplicated for the different boresight directions $a$ and $d$. As a result, the fainter star $a$ is discarded from the boresight directions. Note that $a$ may be included as an adjacent star for other boresight directions. By this procedure, a large number of fainter stars is eliminated from the reference catalog, and stars are distributed evenly through the whole celestial sphere, as shown in Figure. 4. After eliminating these multiplications, $L$ boresight directions remain. An $L \times 4$ index matrix is generated, of which the first column is the index of the brightest adjacent star for each boresight direction and remaining columns are the indexes of the adjacent stars in increasing order in magnitude. Each row of indexes contains indexes of 4 brightest stars. From the index matrix, $M$ guide stars are extracted. Next, pre-calculate the singular values of the 4-column-vector matrix for each boresight direction. Then, an $L \times 3$ floating-point array is required. Total storage requirement is shown in Table. 1. Although the mission catalog has almost evenly distributed guide stars, it can still experience lack of boresight direction, as shown in Figure. 5. When a star camera looks at the celestial part encircled by $A$, the FOV has the minimum requirement of stars; but the mission catalog may not have the corresponding set, because there are no boresight directions having such adjacent stars. This problem can be resolved using the initial reference 
catalog with a larger magnitude limit, but there is still no way to obtain continuous boresight directions.

The pattern recognition process is simple and straightforward. When an image is acquired, the measurement stars are sorted by their instrument magnitude. Consider a measurement star (starting with the brightest star) as boresight direction, and choose 4 brighter stars within the circular FOV. After SVD, singular values are compared with those in the mission catalog. It is possible that multiple identification happens, since measurement noise may cause ambiguity. For these identified subsets, an additional filter is applied from Eq. (15). The direction of each right singular vector from the measurement matrix is compared with that from the identified subsets. The possibility of mismatching approaches to zero. If pattern recognition fails for the first boresight direction, repeat this process with the next boresight direction. Once pattern recognition is accomplished, attitude is estimated by Eq. (24). To confirm success of pattern recognition and attitude estimation, pattern recognition is repeated for subsequent images, and the closeness between the current attitude estimate and previous attitude estimates is tested.

Table. 1 Storage Requirement of Mission Catalog

\begin{tabular}{|c|c|}
\hline Content & Size \\
\hline Guide Star & $M \times 3$ floating point array $(5761 \times 3)$ \\
\hline Index Matrix & $L \times 4$ integer array $(11157 \times 4)$ \\
\hline Singular Values & $L \times 3$ floating point array $(11157 \times 3)$ \\
\hline Total & 484 Kbytes \\
\hline
\end{tabular}

\section{Simulation}

SVD pattern recognition method is applied to Night-Sky images without a-priori attitude information. Night-Sky images are generated by slewing the star camera, as shown in Figure. 6. Using the initializing method developed in this paper, pattern recognition and attitude estimation is performed for 10 subsequent images, and results are 
summarized in Table. 2. For the first 7 images, pattern recognitions are successfully performed. Although some of the images have multiple identifications (image 4 and 6), estimated attitudes in 3-1-3 Euler angles are consistent. Since these images are obtained from modest slewing motion of the star camera, identified attitudes are supposed to be close to each other. The result illustrates this fact. The pattern recognition failed at image 8, 9, and 10. These failures were caused by magnitude ranking error, lack of measurements, and, most likely, lack of boresight direction. However, the advantage of this method is in the low possibility of mismatching by double-checking of the singular values and singular vectors. Note that the failure of pattern recognition is more desirable than mismatching.

Table. 2 Pattern Recognition and Attitude Estimation for Night-Sky Images

\begin{tabular}{|c|c|c|c|c|}
\hline & Pattern Recognition & \multicolumn{3}{|c|}{$3-1-3$ Euler Angles (radian) } \\
\hline Image 1 & 1 & 2.5780 & 1.1583 & 3.1338 \\
\hline Image 2 & 1 & 2.6128 & 1.1583 & 3.1356 \\
\hline Image 3 & 1 & 2.6383 & 1.1587 & 3.1364 \\
\hline \multirow{2}{*}{ Image 4 } & \multirow{2}{*}{2} & 2.6872 & 1.1590 & 3.1374 \\
\cline { 2 - 5 } & \multirow{2}{*}{2} & 2.6873 & 1.1589 & 3.1387 \\
\hline Image 5 & 2.7133 & 1.1589 & 3.1410 \\
\hline \multirow{2}{*}{ Image 6 } & 1 & 2.7439 & 1.1536 & 3.1387 \\
\hline Image 7 & 0 & 2.7408 & 1.1593 & 3.1390 \\
\hline Image 8 & 0 & 2.7937 & 1.1596 & 3.1399 \\
\hline Image 9 & 0 & N/A & \\
\hline Image 10 & 0 & N/A & \\
\hline
\end{tabular}

\section{Conclusion}

A new strategy for star pattern recognition and attitude estimation has been developed by utilizing SVD of a measurement vector matrix and reference vector matrix. The advantage of the proposed method is that the pattern recognition is extremely fast, since 
only three singular values are compared, no matter how many vectors are considered. This is desired for the lost-in-space case without any a-priori attitude estimation. The other advantage of this method is that the pattern recognition and the attitude estimation can be performed simultaneously, or individual star pairing is not necessary. Moreover, the algorithm yields optimal attitude estimate. The mission catalog contains the direction cosines of each guide star, the star index, and the singular values for each boresight direction. The size of the mission catalog is relatively small (under 500Kbytes). Further study, including the quantitative comparison of the proposed method with other initialization algorithms, will be followed by this paper.

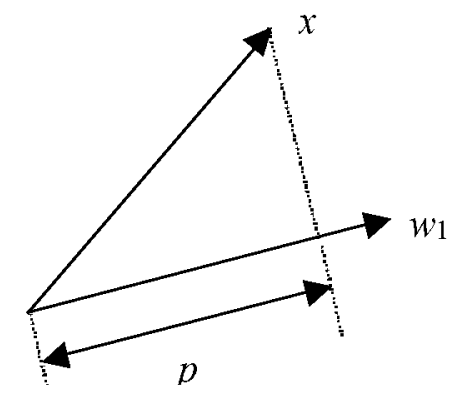

Figure. 1 Projection $x$ onto $w_{1}$

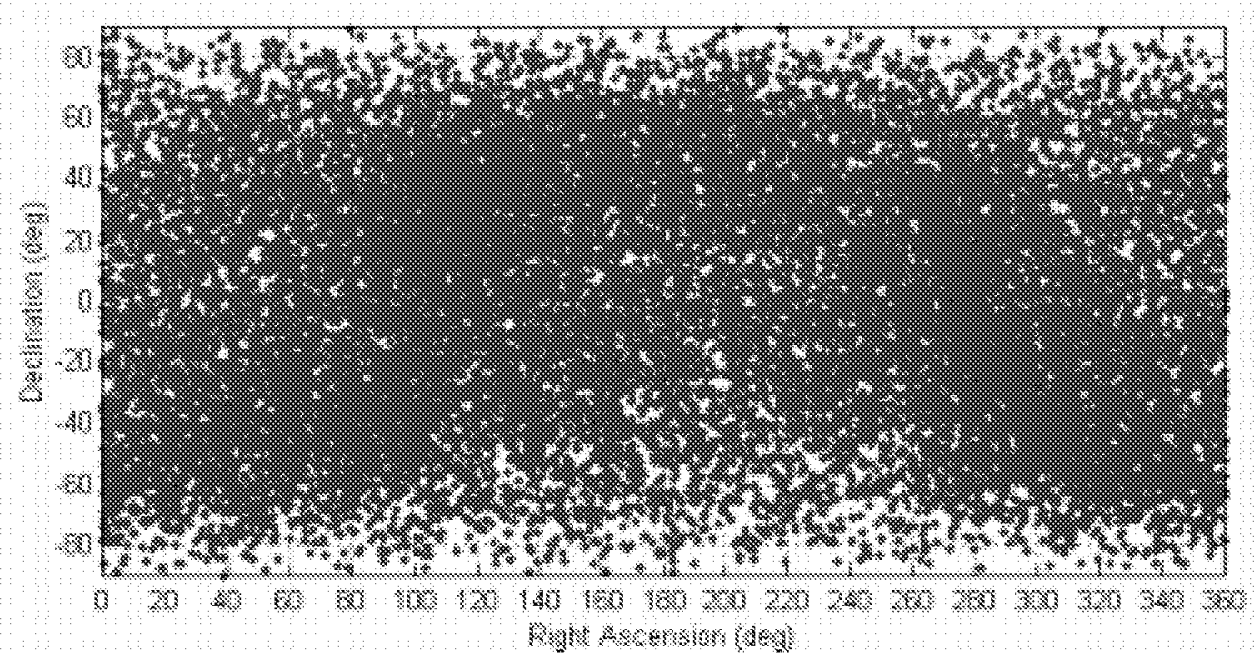

Figure. 2 Initial Reference Catalog Star Distribution 


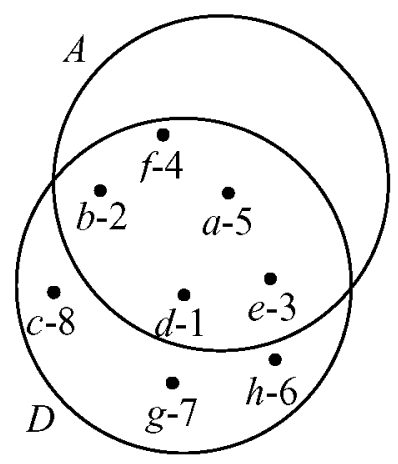

Figure. 3 Mission Catalog Generation

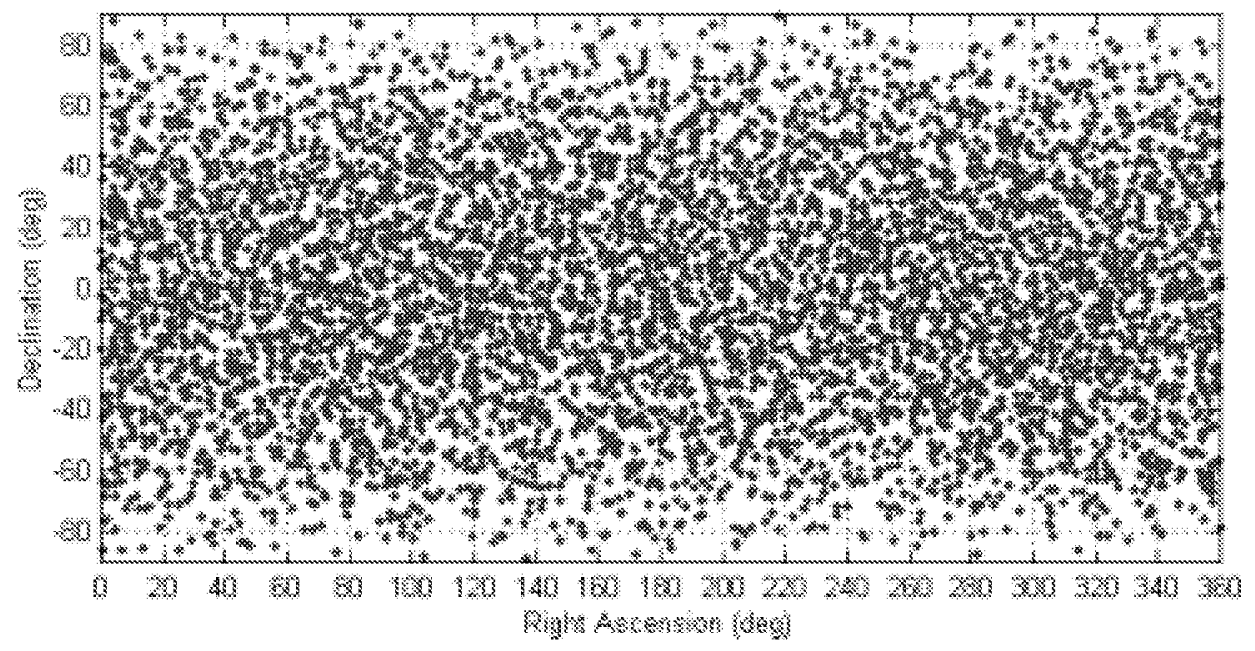

Figure. 4 Mission Catalog Guide Star Distribution

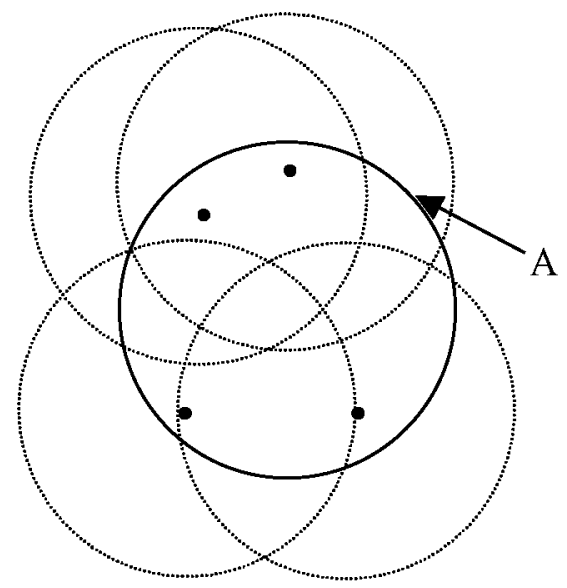

Figure. 5 Lack of Boresight Directions 


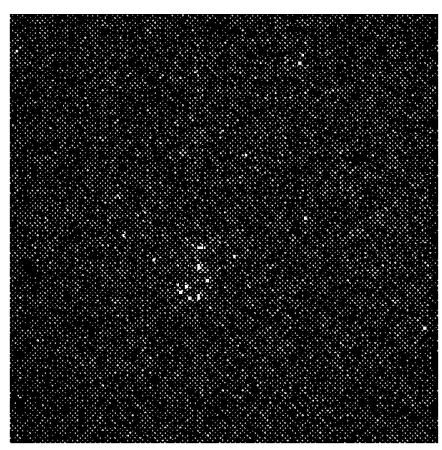

Image 1

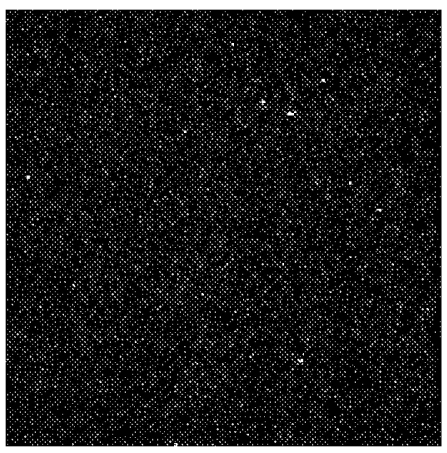

Image 4

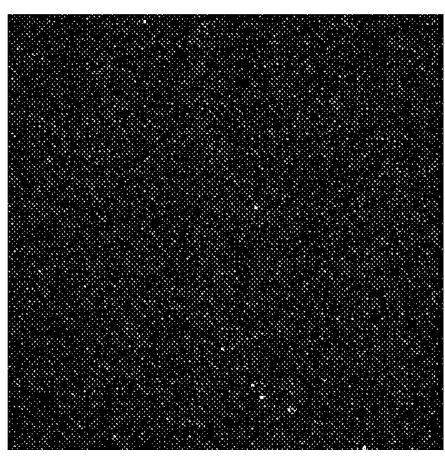

Image 7

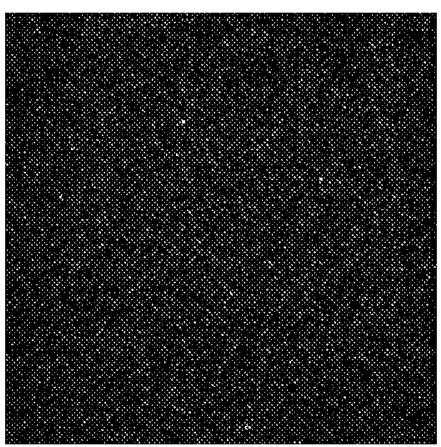

Image 10

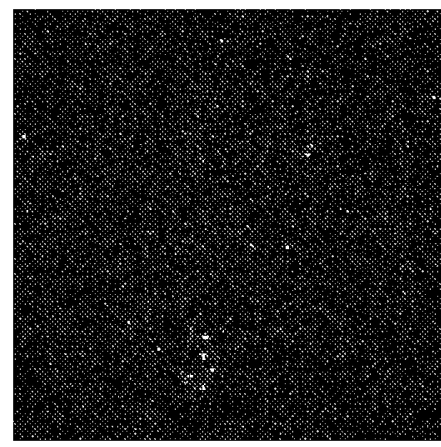

Image 2

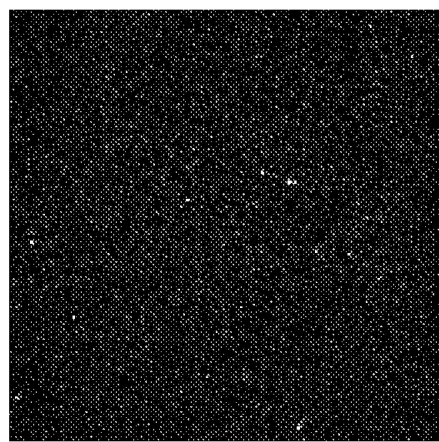

Image 5

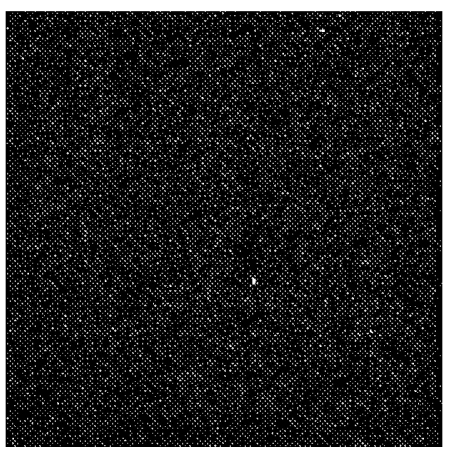

Image 8

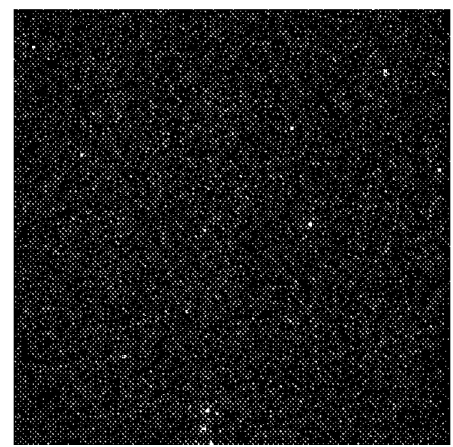

Image 3

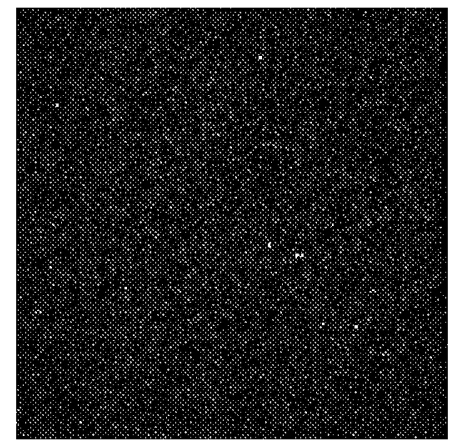

Image 6

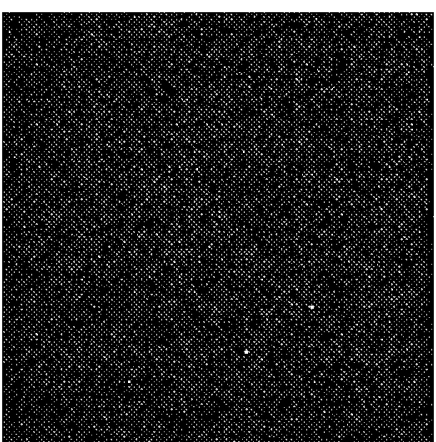

Image 9

Figure. 6 Night-Sky Images 


\section{Reference}

1. Junkins, J. L., White III, C. C., Turner, J. D., "Star Pattern Recognition for Real Time Attitude Determination," The Journal of the Astronautical Sciences, Vol. XXV, No. 3, pp. 251-270, Jul.-Sep. 1977, pp. 251-271.

2. Groth, E. J., "A Pattern-Matching Algorithm For Two-Dimensional Coordinate Lists," The Astronomical Journal, Vol. 91, No. 5, May 1986, pp. 1244-1248.

3. Van Bezooijen, R. W. H., "Success Potential of Automated Star pattern Recognition," ALAA 24th Aerospace Sciences Meeting, Jan. 1986/Reno, Nevada, AIAA-86-0254.

4. Sheela, B. V., and Shekhar, C., "New Star Identification Technique for Attitude Control," Journal of Guidance, Control, and Dynamics, Vol. 14, No. 2, Mar.-Apr. 1991, pp. 477-480.

5. Kosik, J. C., "Star Pattern Identification Aboard an Inertially Stabilized Spacecraft," Journal of Guidance and Control, Vol. 14, No. 2, Mar.-Apr. 1991, 230-235.

6. Liebe, C. C., "Pattern Recognition of Star Constellations for Spacecraft Applications," IEEE AES Magazine, Jun. 1992, pp. 34-41.

7. Udomkesmalee, S., Alexander, J. W., and Tolivar, A. F., "Stochastic Star Identification," Journal of Guidance, Control, and Dynamics, Vol. 17, No. 6, Nov.Dec. 1994, 1283-1286.

8. Mortari, D., "Search-Less Algorithm for Star Pattern Recognition," The Journal of the Astronautical Sciences, Vol. 45, No. 2, Apr.-Jun. 1997, pp. 179-194.

9. Silverman, E. K., "Autonomous Navigation Recovery for Fine Pointing Low Earth Orbiting Spacecraft," Ph. D. Dissertation, George Washington University, 1998.

10. Gottlieb, D. M., "Star Identification Techniques," Spacecraft Attitude Determination and Control, J. R. Wertz (editor), Kluwer Academic Publishers, Dordrecht, The Netherlands, 1978, pp. 259-266.

11. Keat, J., "Analysis of Least-Squares Attitude Determination Routine DOAOP," CSC Report CSC/TM-77/6034, February 1977.

12. Lerner, Gerald M., “Three-Axis Attitude Determination," in Spacecraft Attitude Determination and Control, ed. by James R. Wertz, Dordrecht, Holland, D. Reidel, 1978. 
13. Markley, F. Landis, "Parameterizations of the Attitude," in Spacecraft Attitude Determination and Control, ed. by James R. Wertz, Dordrecht, Holland, D. Reidel, 1978.

14. Shuster, Malcolm D., "A Survey of Attitude Representations," Journal of the Astronautical Sciences, Vol. 41, No. 4, October-December 1993, pp. 439-517.

15. Markley, F. L., and Mortari, D., "How to Estimate Attitude from Vector Observations," AAS 99-427, AAS/AIAA Astrodynamics Specialist Conference, Girdwood, Alaska, August 16-19, 1999.

16. Markley, F. L., "Attitude Determination Using Vector Observations and the Singular Value Decomposition," AAS Paper 87-490, AAS/AIAA Astrodynamics Specialist Conference, Kalispell, MT, August 1987, and Journal of the Astronautical Sciences, Vol. 36, No. 3, July-Sept. 1988, pp. 245-258.

17. Farrell, J. L. and J. C. Stuelpnagel, "A Least Squares Estimate of Spacecraft Attitude," SIAM Review, Vol. 8, No. 3, July 1966, pp. 384-386.

18. Strang, G., Linear Algebra and Its Applications, Harbourt Brace Jovanovich, 1988. 


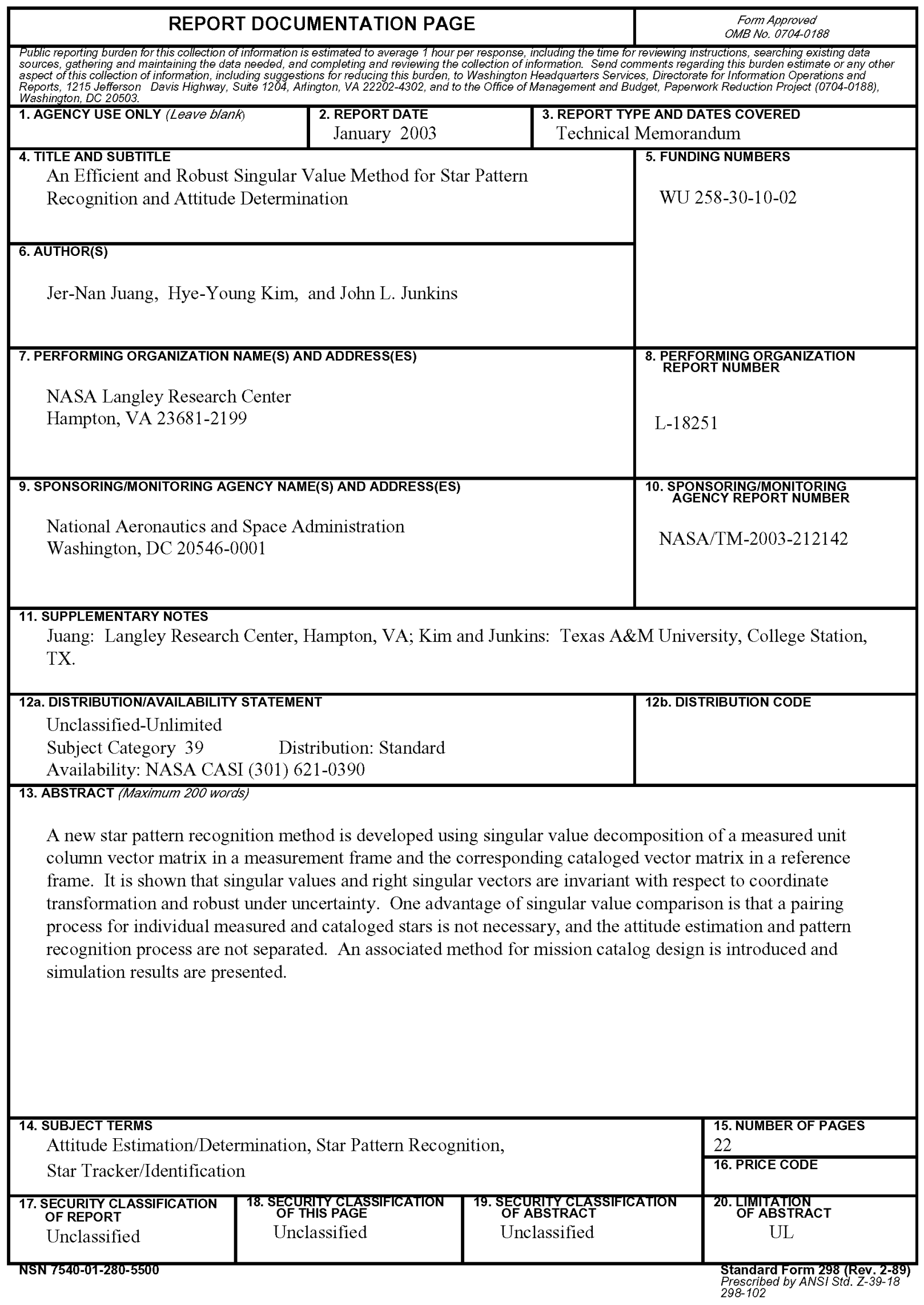

